\title{
Chiral Ward identities for Dirac eigenmodes with staggered fermions
}

\author{
Hwancheol Jeong*, Sunghee Kim, Weonjong Lee, and Jeonghwan Pak \\ Lattice Gauge Theory Research Center, CTP, and FPRD, \\ Department of Physics and Astronomy, \\ Seoul National University, Seoul 08826, South Korea \\ E-mail: sonchacegmail.com, wleedsnu.ac.kr
}

\section{Chulwoo Jung}

Physics Department, Brookhaven National Laboratory, Upton, NY 11973, USA

E-mail: chulwoo@bnl.gov

\section{SWME Collaboration}

We study chiral properties of eigenvalue spectrum for staggered quarks. We present a new method to identify would-be zero modes and nonzero modes using their symmetry and chiral properties. Here, we review the traditional method with HYP improved staggered quarks, and extend it to a completely new method which uses the chiral Ward identities and leakage patterns to achieve the goal.

Wuhan, China

\footnotetext{
*Speaker.
} 


\begin{tabular}{r|l}
\hline \hline parameter & value \\
\hline gluon action & tree level Symanzik [6-8] \\
$\beta$ & 5.0 \\
tadpole improvement & yes \\
geometry & $20^{4}$ \\
$a$ & $0.077 \mathrm{fm}$ \\
\hline valence quarks & HYP staggered fermions [9-11] \\
$N_{f}$ & 0 (quenched QCD) \\
\hline \hline
\end{tabular}

Table 1: Input parameters for the numerical study. For more details, refer to Ref. [12].

\section{Introduction}

Here, we present recent progress in understanding chiral properties of staggered Dirac eigenmodes based on our previous works in Refs. [1,2].

\section{Eigenvalues of Dirac operators with staggered fermions}

Let us consider Dirac operator $D_{s}$ for staggered fermions. Since $D_{s}$ is anti-Hermitian, eigenvalues of $D_{s}$ are purely imaginary or zero:

$$
D_{s}\left|f_{\lambda}\right\rangle=i \lambda\left|f_{\lambda}\right\rangle
$$

where $\lambda$ is real, and $\left|f_{\lambda}\right\rangle$ is an eigenvector with its eigenvalue $i \lambda$.

$D_{s}$ also anti-commutes with the operator $\Gamma_{\varepsilon}=\left[\gamma_{5} \otimes \xi_{5}\right]$ which is the generator for $\mathrm{U}(1)_{\mathrm{A}}$ symmetry. Here, we adopt the same notation as in Ref. [1]. Since $\Gamma_{\varepsilon}$ anti-commutes with $D_{s}$, one can show that an eigenstate $\left|f_{\lambda}\right\rangle$ with $\lambda \neq 0$ has its partner state $\left|f_{-\lambda}\right\rangle$ with eigenvalue $-i \lambda$ [1]. The partner eigenvector $\left|f_{-\lambda}\right\rangle$ can be obtained by applying $\Gamma_{\varepsilon}$ to the eigenvector $\left|f_{+\lambda}\right\rangle$ with phase difference:

$$
\Gamma_{\varepsilon}\left|f_{+\lambda}\right\rangle=e^{+i \theta}\left|f_{-\lambda}\right\rangle, \quad \Gamma_{\varepsilon}\left|f_{-\lambda}\right\rangle=e^{-i \theta}\left|f_{+\lambda}\right\rangle .
$$

Here, the phase $\theta$ is real, and also turns out to be arbitrary [1].

In practice, we do not calculate eigenvalues of $D_{s}$ directly. Instead, we use a Hermitian and positive semi-definite operator $D_{s}^{\dagger} D_{s}$, which satisfies

$$
D_{s}^{\dagger} D_{s}\left|g_{\lambda^{2}}\right\rangle=\lambda^{2}\left|g_{\lambda^{2}}\right\rangle .
$$

The corresponding eigenvectors $\left|f_{ \pm \lambda}\right\rangle$ are obtained by decomposing the eigenvector $\left|g_{\lambda^{2}}\right\rangle$ using the projection operators as in Ref. [1]. Since $D_{s}^{\dagger} D_{s}$ is Hermitian, one can make use of Lanczos algorithm [3] to calculate its eigenvalues and eigenvectors. Here, we use the implicitly restarted Lanczos [4] with acceleration by Chebyshev polynomial [5].

All the numerical calculations are performed on the gauge ensemble described in Table 1. We use HYP staggered fermions as valence quarks which reduce the taste-breaking for staggered fermions, and thus show improved chiral behaviors [12-15].

Meanwhile, the index theorem [16] states that

$$
Q_{t}=n_{-}-n_{+}, \quad Q_{t}=\frac{1}{32 \pi^{2}} \int_{E} d^{4} x \varepsilon_{\alpha \beta \mu \nu} \operatorname{Tr}\left(F_{\alpha \beta} F_{\mu \nu}\right)
$$



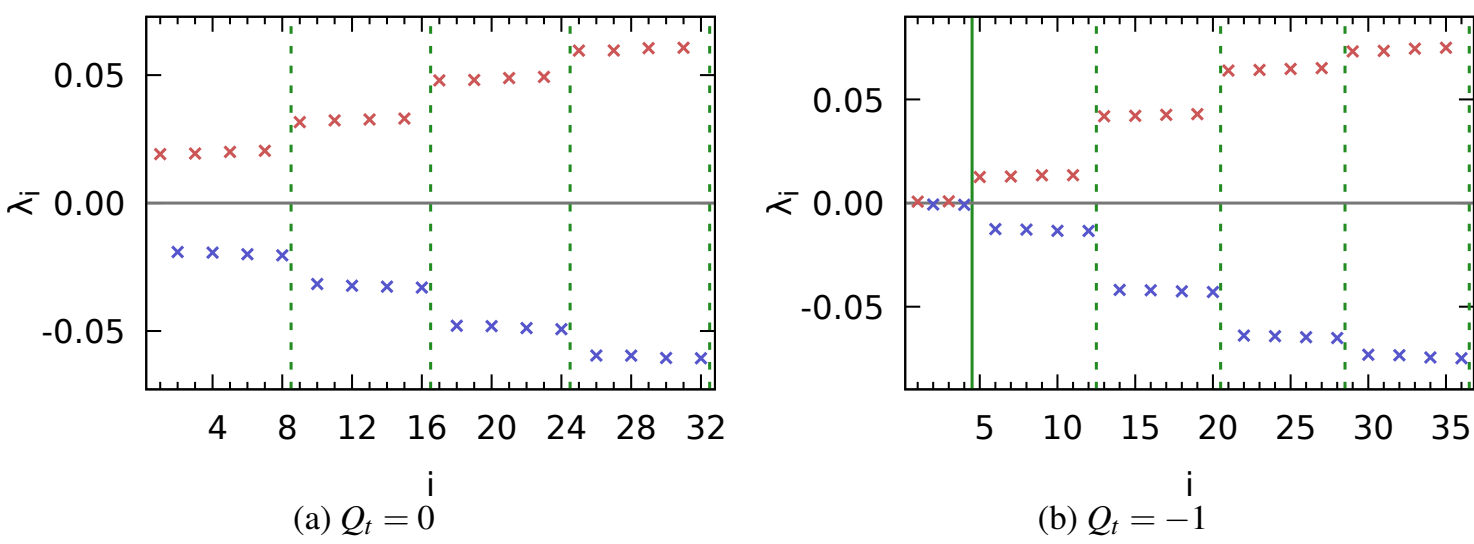

Figure 1: Eigenvalue spectra of staggered Dirac operator on gauge configurations with $Q_{t}=0$ and $Q_{t}=-1$. Here, $i$ represents an index of eigenvalue $\lambda_{i}$.

where $Q_{t}$ is the topological charge [17], the subscript ${ }_{E}$ represents the Euclidean space, and $n_{+}\left(n_{-}\right)$ is the number of zero modes with right-handed (left-handed) helicity. In the continuum, Eq. (2.4) indeed comes from the axial Ward identity [2]. For staggered fermions, a similar relation holds but four-fold degeneracy which comes from the approximate $\mathrm{SU}(4)$ taste symmetry should be counted [1]:

$$
Q_{t}=\frac{1}{4}\left(n_{-}^{s}-n_{+}^{s}\right),
$$

where $n_{ \pm}^{s}$ represent the number of zero modes with right-handed (+) and left-handed (-) helicities for staggered quarks. Here, $n_{ \pm}^{s}$ must be multiples of four due to the taste symmetry.

\section{Eigenvalue spectrum}

In Fig. 1, we present tens of low-lying eigenvalues of the Dirac operator with HYP staggered quarks on gauge configurations with topological charges $Q_{t}=0,-1$. Here, we measure $Q_{t}$ using the $Q(5 \mathrm{Li})$ operator defined in Ref. [18, 19] after $10 \sim 30$ iterations of the APE smearing with $\alpha=0.45$ [20-22]. In the plot, eigenvalues are sorted in ascending order of their absolute values $\left|\lambda_{i}\right|$. Here, we assign the index ( $\left.2 n\right)$ of the eigenvalue such that it satisfies $\lambda_{2 n}=-\lambda_{2 n-1}$. Even the would-be zero modes have tiny but nonzero values of $\lambda_{i}$ at finite lattice spacing $a \neq 0$. Hence, each eigenvalue has its parity partner with opposite sign even though it belongs to the would-be zero modes. The solid green line for $Q_{t}=-1$ is drawn at the boarder between the would-be zero modes and the nonzero modes.

For would-be zero modes, their eigenvalues are exactly zero in the continuum limit, and so they are their own parity partners by themselves. The number of the would be zero modes must be multiple of four since the SU(4) taste symmetry is exactly conserved in the continuum. In Fig. 1b, one can see the would-be zero modes appear with four-fold degeneracy. For nonzero modes, one eigenvalue must have four-fold degeneracy due to the $\mathrm{SU}(4)$ taste symmetry in the continuum, and its $\mathrm{U}(1)_{\text {A }}$ parity partner should have the same four-fold degeneracy. Hence, for each nonzero eigenvalue, it has a set of eight-fold degeneracy due to the exact $\mathrm{U}(1)_{\mathrm{A}}$ symmetry on the lattice and the SU(4) taste symmetry in the continuum. In Fig. 1, one can see the nonzero modes show up with eight-fold degeneracy. 


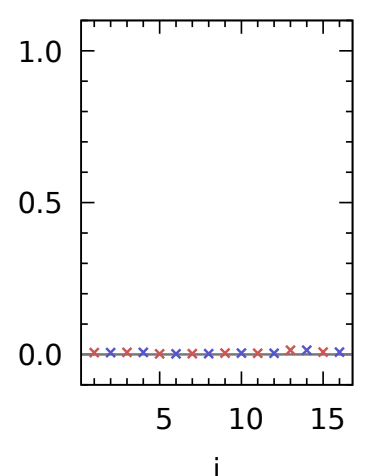

(a) $Q_{t}=0$

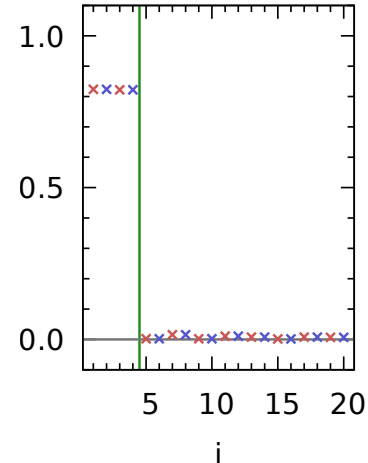

(b) $Q_{t}=-1$

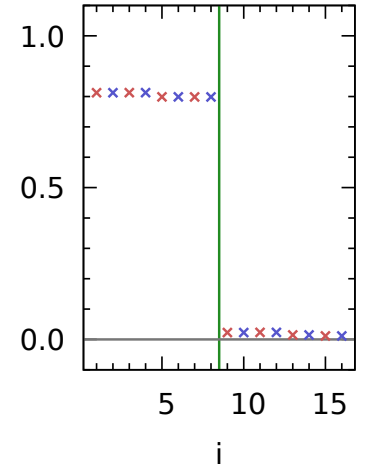

(c) $Q_{t}=-2$

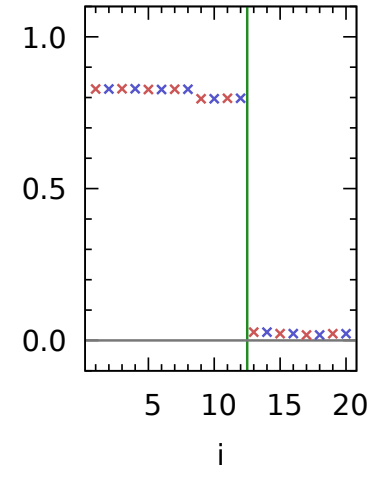

(d) $Q_{t}=-3$

Figure 2: $\Gamma_{5}\left(\lambda_{i}\right)$ for various topological charges. Here, $i$ represents an index of eigenvalue $\lambda_{i}$.

\section{Chirality for staggered fermions}

Let us consider three chirality operators: $\Gamma_{\varepsilon}, \Gamma_{5}$, and $\Xi_{5}$ defined as

$$
\Gamma_{\varepsilon} \equiv\left[\gamma_{5} \otimes \xi_{5}\right], \quad \Gamma_{5} \equiv\left[\gamma_{5} \otimes \mathbb{1}\right], \quad \Xi_{5} \equiv\left[\mathbb{1} \otimes \xi_{5}\right]
$$

$\Gamma_{\varepsilon}$ represents a chirality of the conserved $\mathrm{U}(1)_{\mathrm{A}}$ symmetry for staggered fermions. A taste singlet operator $\Gamma_{5}$ corresponds to the generator for the anomalous $\mathrm{U}(1)_{\mathrm{A}}^{\text {anom }}$ symmetry in the continuum. Similarly, $\Xi_{5}$ represents the parity partner for the chirality operator $\Gamma_{5}$.

The $\Gamma_{\varepsilon}, \Gamma_{5}$, and $\Xi_{5}$ operators satisfy the same relations as the continuum chirality operator $\gamma_{5}$ as follows,

$$
(\Gamma)^{2 n+1}=\Gamma, \quad(\Gamma)^{2 n}=\mathbb{1},
$$

where $\Gamma \in\left\{\Gamma_{\varepsilon}, \Gamma_{5}, \Xi_{5}\right\}$. Furthermore, they are related to each other by

$$
\Gamma_{\varepsilon}=\Gamma_{5} \Xi_{5} ; \quad \Gamma_{5}=\Xi_{5} \Gamma_{\varepsilon} ; \quad \Xi_{5}=\Gamma_{5} \Gamma_{\varepsilon}
$$

These properties insure that they are the best choice to examine the chiral symmetry for staggered fermions.

Let us define the chirality as

$$
\Gamma_{5}(\alpha, \beta) \equiv\left\langle f_{\alpha}\left|\left[\gamma_{5} \otimes \mathbb{1}\right]\right| f_{\beta}\right\rangle, \quad \Gamma_{5}\left(\lambda_{i}\right) \equiv \Gamma_{5}\left(\lambda_{i}, \lambda_{i}\right)
$$

In Fig. 2, we measure the chirality $\Gamma_{5}\left(\lambda_{i}\right)$ for topological charges $Q_{t}=0,-1,-2,-3$, respectively. Comparing with Fig. 1, the would-be zero modes has a non-trivial chirality around 0.8 in magnitude, while nonzero modes have values of $\Gamma_{5}\left(\lambda_{i}\right)$ close to zero. Consequently, would-be zero modes are manifestly distinguishable from nonzero modes by the $\Gamma_{5}$ chirality as shown in Ref. [12-14]. The magnitudes of the chirality for would-be zero modes are somewhat smaller than one, the continuum expectation value. It is because the $\Gamma_{5}$ operator is not conserved at $a \neq 0$ and receives a finite renormalization on the lattice. 


\begin{tabular}{cc}
\hline \hline parameter & value \\
\hline$\left|\Gamma_{5}\left(\lambda_{1}, \lambda_{1}\right)\right|$ & 0.8238257 \\
$\left|\Xi_{5}\left(\lambda_{2}, \lambda_{1}\right)\right|$ & 0.8238257 \\
$\left|\Xi_{5}\left(\lambda_{1}, \lambda_{2}\right)\right|$ & 0.8238257 \\
$\left|\Gamma_{5}\left(\lambda_{2}, \lambda_{2}\right)\right|$ & 0.8238257 \\
\hline \hline
\end{tabular}

(a) Diagonal WI

\begin{tabular}{cc|cc}
\hline \hline parameter & value & parameter & value \\
\hline$\left|\Gamma_{5}\left(\lambda_{75}, \lambda_{70}\right)\right|$ & 0.5008622 & $\left|\Gamma_{5}\left(\lambda_{70}, \lambda_{75}\right)\right|$ & 0.5008622 \\
$\left|\Xi_{5}\left(\lambda_{69}, \lambda_{75}\right)\right|$ & 0.5008622 & $\left|\Xi_{5}\left(\lambda_{75}, \lambda_{69}\right)\right|$ & 0.5008622 \\
$\left|\Xi_{5}\left(\lambda_{70}, \lambda_{76}\right)\right|$ & 0.5008622 & $\left|\Xi_{5}\left(\lambda_{76}, \lambda_{70}\right)\right|$ & 0.5008622 \\
$\left|\Gamma_{5}\left(\lambda_{69}, \lambda_{76}\right)\right|$ & 0.5008622 & $\left|\Gamma_{5}\left(\lambda_{76}, \lambda_{69}\right)\right|$ & 0.5008622 \\
\hline \hline
\end{tabular}

(b) Off-diagonal WI

Table 2: Numerical demonstration of chiral Ward identity (WI) in Eq. (5.8). Here, $\lambda_{2}=-\lambda_{1}$, $\lambda_{70}=-\lambda_{69}, \lambda_{76}=-\lambda_{75}$.

\section{Chiral Ward identity}

Rewriting Eqs. (2.2) by implementing Eq. (4.2) and Eq. (4.3), we obtain the following chiral Ward identities for staggered fermions:

$$
\Gamma_{5}\left|f_{+\lambda}\right\rangle=e^{+i \theta} \Xi_{5}\left|f_{-\lambda}\right\rangle, \quad \Gamma_{5}\left|f_{-\lambda}\right\rangle=e^{-i \theta} \Xi_{5}\left|f_{+\lambda}\right\rangle .
$$

Let us define the chirality matrix elements sandwiched between the two eigenvectors as

$$
\begin{aligned}
& \Gamma_{\varepsilon}(\alpha, \beta) \equiv\left\langle f_{\alpha}\left|\Gamma_{\varepsilon}\right| f_{\beta}\right\rangle=\left\langle f_{\alpha}\left|\left[\gamma_{5} \otimes \xi_{5}\right]\right| f_{\beta}\right\rangle \\
& \Gamma_{5}(\alpha, \beta) \equiv\left\langle f_{\alpha}\left|\Gamma_{5}\right| f_{\beta}\right\rangle=\left\langle f_{\alpha}\left|\left[\gamma_{5} \otimes \mathbb{1}\right]\right| f_{\beta}\right\rangle \\
& \Xi_{5}(\alpha, \beta) \equiv\left\langle f_{\alpha}\left|\Xi_{5}\right| f_{\beta}\right\rangle=\left\langle f_{\alpha}\left|\left[\mathbb{1} \otimes \xi_{5}\right]\right| f_{\beta}\right\rangle .
\end{aligned}
$$

Using the Ward identity of Eqs. (5.1), we rewrite the chirality matrix elements as follows,

$$
\begin{array}{ll}
\Gamma_{5}(\alpha,+\beta)=e^{+i \theta_{\beta}} \Xi_{5}(\alpha,-\beta), & \Gamma_{5}(\alpha,-\beta)=e^{-i \theta_{\beta}} \Xi_{5}(\alpha,+\beta), \\
\Gamma_{5}(+\alpha, \beta)=e^{-i \theta_{\alpha}} \Xi_{5}(-\alpha, \beta), & \Gamma_{5}(-\alpha, \beta)=e^{+i \theta_{\alpha}} \Xi_{5}(+\alpha, \beta) .
\end{array}
$$

If we take the norm of them, then

$$
\left|\Gamma_{5}(\alpha, \beta)\right|=\left|\Xi_{5}(\alpha,-\beta)\right|=\left|\Xi_{5}(-\alpha, \beta)\right|=\left|\Gamma_{5}(-\alpha,-\beta)\right| .
$$

In addition, the Hermiticity insures interchanging $\alpha$ and $\beta$, which provides the final form of the Ward identities:

$$
\begin{aligned}
\left|\Gamma_{5}(\alpha, \beta)\right| & =\left|\Xi_{5}(\alpha,-\beta)\right|=\left|\Xi_{5}(-\alpha, \beta)\right|=\left|\Gamma_{5}(-\alpha,-\beta)\right| \\
& =\left|\Gamma_{5}(\beta, \alpha)\right|=\left|\Xi_{5}(\beta,-\alpha)\right|=\left|\Xi_{5}(-\beta, \alpha)\right|=\left|\Gamma_{5}(-\beta,-\alpha)\right| .
\end{aligned}
$$

Table 2 shows how the chiral Ward identities of Eq. (5.8) works in our numerical study. Here, it confirms that they are valid within our numerical precision.

\section{Leakage of chirality}

Here, we focus on off-diagonal elements $(\alpha \neq \beta)$ of chirality $\Gamma_{5}(\alpha, \beta)$ and $\Xi_{5}(\alpha, \beta)$. We are interested in how much of the chirality of an eigenmode leaks into other eigenmodes. Traditionally, the diagonal elements of chirality $\Gamma_{5}\left(\lambda_{i}\right)$ were measured and studied as in Ref. [12-14]. Here, 


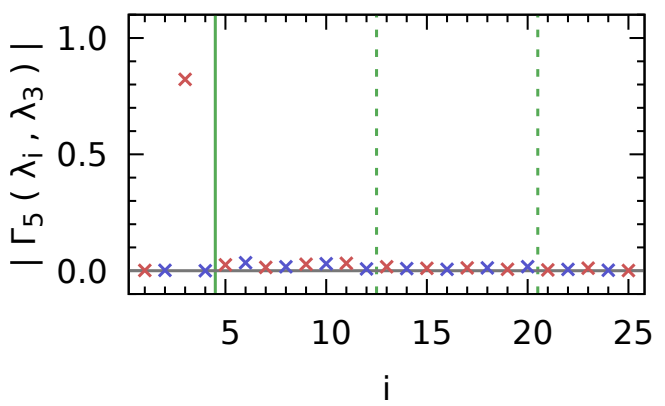

(a) $Q_{t}=-1$

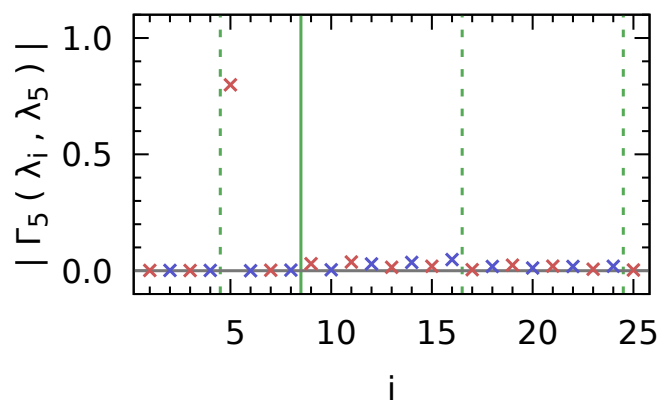

(b) $Q_{t}=-2$

Figure 3: Leakage patterns of would-be zero modes for the $\Gamma_{5}$ operator.

we study on the off-diagonal elements of chirality $\Gamma_{5}(\alpha, \beta)$, and $\Xi_{5}(\alpha, \beta)$ with $\alpha \neq \beta$. In the continuum, the $\mathrm{SU}(4)$ taste symmetry is respected. The net consequence of the conserved taste symmetry is that each nonzero eigenvalue has eight-fold degeneracy, and these eight degenerate eigenmodes will mix with one another within the eight-fold degenerate members. In other words, if $|\alpha| \neq|\beta|$, then $\Gamma_{5}(\alpha, \beta)=\Xi_{5}(\alpha, \beta)=0$ in the continuum $(a=0)$ thanks to the SU(4) taste symmetry. However, at finite lattice $(a \neq 0)$, the SU(4) taste symmetry is not exact, but mostly respected near the continuum. Hence, the leakage from one set of the eight-fold degeneracy to other set of eight-fold degeneracy will be very small near the continuum $(a \approx 0)$. Therefore, it will be very interesting to study this leakage pattern in the chirality measurement. Fig. 3 shows leakage patterns of $\Gamma_{5}$ for would-be zero modes. Here, we observe that for $\Gamma_{5}$ there is only one non-trivial signal at the would-be zero mode itself and there is almost no leakage to nearest zero and nonzero eigenmodes.

For nonzero modes, leakage of the $\Gamma_{5}$ operator for an eigenvalue $\lambda_{i}$ is supposed to go into the four-fold parity partners with eigenvalue $-\lambda_{i}$ in the continuum. Near the continuum $(a \approx 0)$, the SU(4) taste symmetry is almost respected and the leakage to eigenmodes outside the eightfold degeneracy is also almost prohibited. This kind of leakage patterns for nonzero eigenmodes are presented in Fig. 4. In Fig. 4a, the leakage of the $\Gamma_{5}$ chirality for the eigenmode $\left|f_{\lambda_{5}}\right\rangle$ goes into the eigenmodes with eigenvalue $-\lambda_{5}:\left|f_{\lambda_{j}}\right\rangle$ with $j=6,8,10,12$, as the theory predicts. In Fig. $4 \mathrm{~b}$, the leakage of the $\Gamma_{5}$ chirality for the eigenmode $\left|f_{\lambda_{135}}\right\rangle$ goes into the eigenmodes with eigenvalue $-\lambda_{135}:\left|f_{\lambda_{j}}\right\rangle$ with $j=134,136,138,140$. Here, we also observe a small effect of the SU(4) taste symmetry breaking in that a small amount of leakage of the $\Gamma_{5}$ chirality for the eigenmode $\left|f_{\lambda_{135}}\right\rangle$ goes into eigenmodes outside of the eight-fold degeneracy such as $\left|f_{\lambda_{j}}\right\rangle$ with $j=126,128,130,132,142,144$.

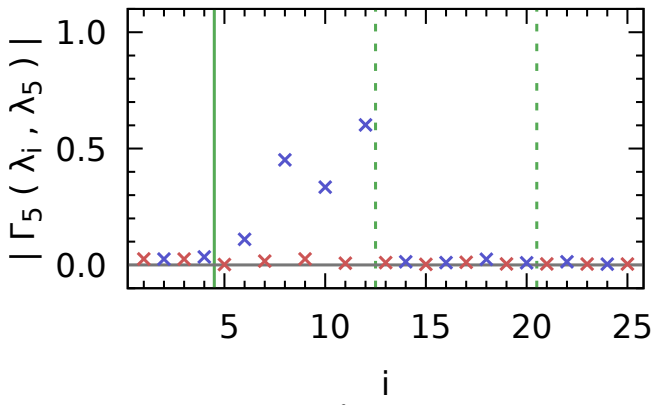

(a) Leakage for $\lambda_{5}$ with $Q_{t}=-1$

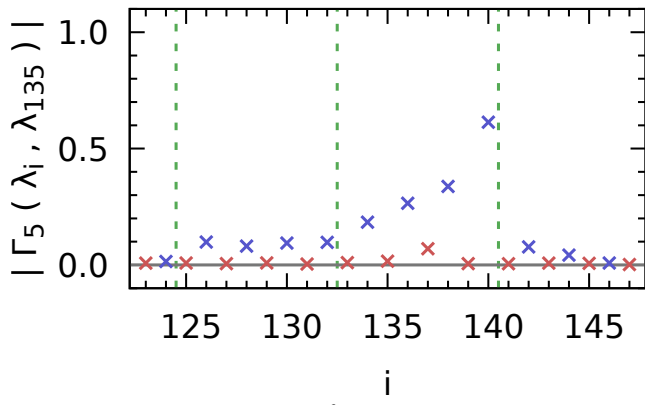

(b) Leakage for $\lambda_{135}$ with $Q_{t}=-1$

Figure 4: Leakage patterns of $\Gamma_{5}$ for nonzero eigenmodes. 


\section{Conclusion}

We have studied $\Gamma_{5}$ and $\Xi_{5}$ chirality for eigenmodes of staggered fermions. Thanks to the Ward identities, $\Gamma_{5}$ chirality is completely correlated with $\Xi_{5}$ chirality. We demonstrate how the leakage patterns of $\Gamma_{5}$ chirality can be used to distinguish zero eigenmodes and nonzero eigenmodes.

\section{Acknowledgments}

We would like to express our sincere gratitude to Eduardo Follana for his kind help. The research of W. Lee is supported by the Mid-Career Research Program (Grant No. NRF-2019R1A2C2085685) of the NRF grant funded by the Korean government (MOE). This work was supported by Seoul National University Research Grant in 2019. W. Lee would like to acknowledge the support from the KISTI supercomputing center through the strategic support program for the supercomputing application research (No. KSC-2017-G2-0009). Computations were carried out on the DAVID cluster at Seoul National University.

\section{References}

[1] H. Jeong, S. Jwa, J. Kim, S. Kim, S. Lee, W. Lee et al., 2017, 1711. 01826.

[2] N. D. Cundy, H. Jeong and W. Lee, PoS LATTICE2015 (2016) 066.

[3] C. Lanczos, J. Res. Natl. Bur. Stand. B Math. Sci. 45 (1950) 255.

[4] R. Lehoucq and D. C. Sorensen, SIAM J. Matrix Anal. Appl 17 (1996) 789.

[5] Y. Saad, Math. Comp. 42 (1984) 567.

[6] M. Luscher and P. Weisz, Commun. Math. Phys. 97 (1985) 59.

[7] M. Luscher and P. Weisz, Phys. Lett. 158B (1985) 250.

[8] M. G. Alford et al., Phys. Lett. B361 (1995) 87 [hep-lat/ 9507010$].$

[9] W.-j. Lee and S. R. Sharpe, Phys. Rev. D66 (2002) 114501 [hep-lat/ 0208018 ].

[10] J. Kim, W. Lee and S. R. Sharpe, Phys. Rev. D81 (2010) 114503 [1004 . 4039 ].

[11] J. Kim, W. Lee and S. R. Sharpe, Phys. Rev. D83 (2011) 094503 [11 02 . 1774].

[12] HPQCD, UKQCD collaboration, Phys. Rev. D72 (2005) 054501 [hep-lat/ 0507011 ].

[13] HPQCD, UKQCD collaboration, Phys. Rev. Lett. 93 (2004) 241601 [hep-lat/ 0406010 ].

[14] E. Follana, A. Hart and C. T. H. Davies, Nucl. Phys. Proc. Suppl. 153 (2006) 106.

[15] T. Bae et al., Phys. Rev. D77 (2008) 094508 [0 801 . 3000].

[16] M. F. Atiyah and I. M. Singer, Bull. Am. Math. Soc. 69 (1969) 422.

[17] CP-PACS collaboration, Phys. Rev. D64 (2001) 114501 [hep-lat/0106010].

[18] P. de Forcrand et al., Nucl. Phys. B499 (1997) 409 [hep-lat/9701012].

[19] P. de Forcrand et al., Nucl. Phys. Proc. Suppl. 47 (1996) 777 [hep-lat/ 9509064 ].

[20] K. Cichy et al., PoS LATTICE2014 (2014) 075 [1411 . 1205].

[21] A. Hasenfratz and C. Nieter, Phys. Lett. B439 (1998) 366 [hep-lat/ 9806026 ].

[22] M. Falcioni, M. L. Paciello, G. Parisi and B. Taglienti, Nucl. Phys. B251 (1985) 624. 